\title{
The rates in complete moment convergence for negatively associated sequences
}

\author{
YUEXU ZHAO*, ZHEYONG QIU, CHUNGUO ZHANG \\ and YEHUA ZHAO \\ Department of mathematics, Hangzhou Dianzi University, Hangzhou 310018, China \\ E-mail: yxzhao@hdu.edu.cn
}

\begin{abstract}
Let $X_{1}, X_{2}, \ldots$ be a strictly stationary and negatively associated sequence of random variables with mean zero and positive, finite variance, set $S_{n}=X_{1}+\cdots+X_{n}$, $M_{n}=\max _{1 \leq k \leq n}\left|S_{k}\right|$. Under appropriate moment conditions, we obtain precise rates in law of the logarithm for the moment convergence of $S_{n}$ and $M_{n}$.
\end{abstract}

Mathematical subject classification: Primary: 60F15; Secondary: 60G50.

Key words: the rates, complete moment convergence, negatively associated sequences.

\section{Introduction and main results}

A finite family of random variables, $X_{1}, X_{2}, \ldots, X_{n}$, is said to be negatively associated if, for every pair of disjoint subsets $T_{1}$ and $T_{2}$ of $\{1,2, \ldots, n\}$,

$$
\operatorname{Cov}\left(f_{1}\left(X_{i}, i \in T_{1}\right), f_{2}\left(X_{j}, j \in T_{2}\right)\right) \leq 0,
$$

whenever $f_{1}$ and $f_{2}$ are coordinatewise increasing and the covariance exists. An infinite family is negatively associated if every finite subfamily is negatively associated. This definition was introduced by Alam and Saxena [1] and JoagDev and Proschan [7], and has found many applications in percolation theory, multivariate statistical analysis and reliability theory [2].

\#CAM-55/09. Received: 11/II/09. Accepted: 18/II/09.

Supported by the Natural Science Foundation of Department of Education of Zhejiang Province(Y200804716)

*Corresponding author. 
Under appropriate conditions, lots of results have been obtained for negatively associated sequences, the central limit theorem (CLT) [13], probability inequalities [15, 17], weak convergence [19, 20], almost sure convergence [12], law of the iterated logarithm (LIL) [16] and complete convergence $[8,9]$, precise rates $[5,21,22]$.

Let $X_{1}, X_{2}, \ldots$ be a sequence of independent and identically distributed (i.i.d.) random variables. Gut and Spătaru [6] obtained a result below.

Theorem A. Suppose that $E X_{1}=0$ and $E X_{1}^{2}=\sigma^{2}<\infty$. Then, for $0 \leq \delta \leq 1$,

$$
\lim _{\epsilon \downarrow 0} \epsilon^{2 \delta+2} \sum_{n=1}^{\infty} \frac{(\log n)^{\delta}}{n} P\left(\left|S_{n}\right| \geq \epsilon \sqrt{n \log n}\right)=\frac{\sigma^{2 \delta+2} E|N|^{2 \delta+2}}{\delta+1} .
$$

where $N$ is a standard normal random variable.

Liu and Lin [11] proved the following theorem for i.i.d. random variables.

Theorem B. Suppose that

$$
E X_{1}=0, E X_{1}^{2}=\sigma^{2} \text { and } E X_{1}^{2}\left(\log ^{+}\left|X_{1}\right|\right)^{\alpha}<\infty .
$$

for $0<\alpha \leq 1$. Then

$$
\lim _{\epsilon \downarrow 0} \epsilon^{2 \alpha} \sum_{n=2}^{\infty} \frac{(\log n)^{\alpha-1}}{n^{2}} E S_{n}^{2} I\left(\left|S_{n}\right| \geq \epsilon \sqrt{n \log n}\right)=\frac{\sigma^{2 \alpha+2}}{\alpha} E|N|^{2 \alpha+2} .
$$

Conversely, if (1.3) is true, then (1.2) holds.

The purpose of the present paper is to investigate precise asymptotics in complete moment convergence, our results not only extend (1.3) to negatively associated sequences, but give a maximal analog of (1.3) and other versions. To formulate our results,we need some extra notation. Let $X_{1}, X_{2}, \ldots$ be strictly stationary and negatively associated random variables, $E X_{1}=0, E X_{1}^{2}<\infty$, $\sigma^{2}=E X_{1}^{2}+2 \sum_{n=2}^{\infty} E X_{1} X_{n}>0$, set $S_{n}=X_{1}+\cdots+X_{n}, M_{n}=\max _{1 \leq k \leq n}\left|S_{k}\right|$, write $\log$ for the natural $\operatorname{logarithm}, \log x=\log _{e}(x \vee e),[z]$ denotes the integer part of $z, C$ stands for a positive constant whose value may be different from line to line. Our results read as follows. 
Theorem 1.1. If $E X_{1}^{2}\left(\log \left|X_{1}\right|\right)^{1-2 / \delta}<\infty$ for any $\delta>2$, then we have

$$
\begin{gathered}
\lim _{\epsilon \downarrow 0} \epsilon^{\delta-2} \sum_{n=2}^{\infty} \frac{1}{n^{2}(\log n)^{2 / \delta}} E S_{n}^{2} I\left(\left|S_{n}\right| \geq \epsilon \sigma \sqrt{n}(\log n)^{1 / \delta}\right) \\
=\frac{\delta(\sqrt{2})^{\delta}}{\sqrt{\pi}(\delta-2)} \Gamma\left(\frac{\delta+1}{2}\right),
\end{gathered}
$$

and

$$
\begin{gathered}
\lim _{\epsilon \downarrow 0} \epsilon^{\delta-2} \sum_{n=2}^{\infty} \frac{1}{n^{2}(\log n)^{2 / \delta}} E M_{n}^{2} I\left(M_{n} \geq \epsilon \sigma \sqrt{n}(\log n)^{1 / \delta}\right) \\
=\frac{2 \delta(\sqrt{2})^{\delta} \Gamma\left(\frac{\delta+1}{2}\right)}{\sqrt{\pi}(\delta-2)} \sum_{n=0}^{\infty} \frac{(-1)^{n}}{(2 n+1)^{2 \delta+2}} .
\end{gathered}
$$

Conversely, if (1.5) is true, then $E X_{1}^{2}\left(\log \left|X_{1}\right|\right)^{1-2 / \delta}<\infty$. Where $\Gamma(\cdot)$ is the Gamma function.

Theorem 1.2. If $E X_{1}^{2}\left(\log \left|X_{1}\right|\right)^{\delta}<\infty$ for any $\delta>0$, then we have

$$
\begin{gathered}
\lim _{\epsilon \downarrow 0} \epsilon^{2 \delta} \sum_{n=2}^{\infty} \frac{(\log n)^{\delta-1}}{n^{2}} E S_{n}^{2} I\left(\left|S_{n}\right| \geq \epsilon \sigma \sqrt{n \log n}\right) \\
=\frac{2^{\delta+1}}{\sqrt{\pi} \delta} \Gamma\left(\delta+\frac{3}{2}\right),
\end{gathered}
$$

and

$$
\begin{gathered}
\lim _{\epsilon \downarrow 0} \epsilon^{2 \delta} \sum_{n=2}^{\infty} \frac{(\log n)^{\delta-1}}{n^{2}} E M_{n}^{2} I\left(M_{n} \geq \epsilon \sigma \sqrt{n \log n}\right) \\
=\frac{2^{\delta+2} \Gamma\left(\delta+\frac{3}{2}\right)}{\sqrt{\pi} \delta} \sum_{n=0}^{\infty} \frac{(-1)^{n}}{(2 n+1)^{2 \delta+2}} .
\end{gathered}
$$

Conversely, if (1.7) is true, then $E X_{1}^{2}\left(\log \left|X_{1}\right|\right)^{\delta}<\infty$.

Without loss of generality, throughout the paper, we will suppose that $\sigma^{2}=1$.

\section{Proof of Theorem 1.1}

In order to verify this result, we first give three elementary but useful lemmas. 
Lemma 2.1. [13]. Let $\left\{X_{n}: n \geq 1\right\}$ be a strictly stationary and negatively associated sequence of random variables with mean zero and

$$
0<\sigma^{2}=E X_{1}^{2}+2 \sum_{n=2}^{\infty} E X_{1} X_{n}<\infty,
$$

then

$$
S_{n} / \sigma \sqrt{n} \stackrel{\mathcal{D}}{\rightarrow} N(0,1) \text { as } n \rightarrow \infty
$$

Lemma 2.2. [18]. Let $\left\{X_{n}: n \geq 1\right\}$ be strictly stationary and negatively associated sequence of random variables, $E X_{1}=\mu, 0<\operatorname{Var} X_{1}=\sigma^{2}<\infty$ and $B^{2}=E X_{1}^{2}+2 \sum_{n=2}^{\infty} E X_{1} X_{n}>0$, set $S_{m}=\sum_{k=1}^{m} X_{k}$, write

$$
W_{n}(t)=\frac{1}{B \sqrt{n}}\left(S_{m}+(n t-m) X_{m+1}-n t \mu\right), m \leq n<m+1,0 \leq t \leq T .
$$

Then

$$
W_{n}(t) \stackrel{\mathcal{D}}{\rightarrow} W(t) \text { in } C[0, T],
$$

where $\{W(t): t \geq 0\}$ is a standard Wiener process and $C[0, T]$ is the usual $C$ space on $[0, T]$.

Lemma 2.3. [10]. Let $\left\{X_{n}: n \geq 1\right\}$ be a negatively associated sequence of random variables with mean zero, $E X_{n}^{2}<\infty$, set $S_{n}=\sum_{k=1}^{n} X_{k}, B_{n}^{2}=$ $\sum_{k=1}^{n} E X_{k}^{2}$. Then, for any $a>0$ and $b>0$, we have

$$
\begin{gathered}
P\left(\max _{1 \leq k \leq n}\left|S_{k}\right| \geq a\right) \leq \\
2 P\left(\max _{1 \leq k \leq n}\left|X_{k}\right| \geq b\right)+2 e \exp \left(\frac{a}{b}-\left(\frac{a}{b}+\frac{B_{n}^{2}}{b^{2}}\right) \log \left(1+\frac{a b}{B_{n}^{2}}\right)\right) .
\end{gathered}
$$

Observe the following formula.

$$
\begin{aligned}
\sum_{n=2}^{\infty} & \frac{1}{n^{2}(\log n)^{2 / \delta}} E S_{n}^{2} I\left(\left|S_{n}\right| \geq \epsilon \sqrt{n}(\log n)^{1 / \delta}\right) \\
= & \epsilon^{2} \sum_{n=2}^{\infty} \frac{1}{n} P\left(\left|S_{n}\right| \geq \epsilon \sqrt{n}(\log n)^{1 / \delta}\right) \\
& +\sum_{n=2}^{\infty} \frac{1}{n^{2}(\log n)^{2 / \delta}} \int_{\epsilon \sqrt{n}(\log n)^{1 / \delta}}^{\infty} 2 x P\left(\left|S_{n}\right| \geq x\right) d x .
\end{aligned}
$$


Similarly, one can obtain the corresponding equation for $M_{n}$. In the rest of this section, we give the following propositions.

Proposition 2.1. We have

$$
\lim _{\epsilon \downarrow 0} \epsilon^{\delta} \sum_{n=2}^{\infty} \frac{1}{n} P\left(|N| \geq \epsilon(\log n)^{1 / \delta}\right)=\frac{(\sqrt{2})^{\delta}}{\sqrt{\pi}} \Gamma\left(\frac{\delta+1}{2}\right),
$$

and

$$
\begin{gathered}
\lim _{\epsilon \downarrow 0} \epsilon^{\delta} \sum_{n=2}^{\infty} \frac{1}{n} P\left(\sup _{0 \leq t \leq 1}|W(t)| \geq \epsilon(\log n)^{1 / \delta}\right) \\
=\frac{2(\sqrt{2})^{\delta} \Gamma\left(\frac{\delta+1}{2}\right)}{\sqrt{\pi}} \sum_{n=0}^{\infty} \frac{(-1)^{n}}{(2 n+1)^{2 \delta+2}},
\end{gathered}
$$

where $N$ is a standard normal random variable and $\{W(t): t \geq 0\}$ is a standard Wiener process.

Proposition 2.2. Under the conditions of Theorem 1.1, we have

$$
\lim _{\epsilon \downarrow 0} \epsilon^{\delta} \sum_{n=2}^{\infty} \frac{1}{n}\left|P\left(\left|S_{n}\right| \geq \epsilon \sqrt{n}(\log n)^{1 / \delta}\right)-P\left(|N| \geq \epsilon(\log n)^{1 / \delta}\right)\right|=0,
$$

and

$$
\begin{aligned}
& \lim _{\epsilon \downarrow 0} \epsilon^{\delta} \sum_{n=2}^{\infty} \frac{1}{n} \mid P\left(M_{n} \geq \epsilon \sqrt{n}(\log n)^{1 / \delta}\right) \\
& \quad-P\left(\sup _{0 \leq t \leq 1}|W(t)| \geq \epsilon(\log n)^{1 / \delta}\right) \mid=0 .
\end{aligned}
$$

Remark 2.1. The proofs of Propositions 2.1 and 2.2 are very standard, so we omit them.

Proposition 2.3. Under the conditions of Theorem 1.1, we have

$$
\begin{gathered}
\lim _{\epsilon \downarrow 0} \epsilon^{\delta-2} \sum_{n=2}^{\infty} \frac{1}{n^{2}(\log n)^{2 / \delta}} \int_{\epsilon \sqrt{n}(\log n)^{1 / \delta}}^{\infty} 2 x P(|N| \geq x / \sqrt{n}) d x \\
=\frac{2(\sqrt{2})^{\delta}}{\sqrt{\pi}(\delta-2)} \Gamma\left(\frac{\delta+1}{2}\right),
\end{gathered}
$$


and

$$
\begin{gathered}
\lim _{\epsilon \downarrow 0} \epsilon^{\delta-2} \sum_{n=2}^{\infty} \frac{1}{n^{2}(\log n)^{2 / \delta}} \int_{\epsilon \sqrt{n}(\log n)^{1 / \delta}}^{\infty} 2 x P\left(\sup _{0 \leq t \leq 1}|W(t)| \geq x / \sqrt{n}\right) d x \\
=\frac{4(\sqrt{2})^{\delta} \Gamma\left(\frac{\delta+1}{2}\right)}{\sqrt{\pi}(\delta-2)} \sum_{n=0}^{\infty} \frac{(-1)^{n}}{(2 n+1)^{2 \delta+2}} .
\end{gathered}
$$

Proof. It follows that

$$
\begin{aligned}
& \lim _{\epsilon \downarrow 0} \epsilon^{\delta-2} \sum_{n=2}^{\infty} \frac{1}{n^{2}(\log n)^{2 / \delta}} \int_{\epsilon \sqrt{n}(\log n)^{1 / \delta}}^{\infty} 2 x P(|N| \geq x / \sqrt{n}) d x \\
& =\lim _{\epsilon \downarrow 0} \epsilon^{\delta-2} \int_{2}^{\infty} \frac{1}{y(\log y)^{2 / \delta}} d y \int_{\epsilon(\log y)^{1 / \delta}}^{\infty} 2 x P(|N| \geq x) d x \\
& =\delta \int_{0}^{\infty} t^{\delta-3} d t \int_{t}^{\infty} 2 x P(|N| \geq x) d x \\
& =\frac{2 \delta}{\delta-2} \int_{0}^{\infty} x^{\delta-1} d x \int_{x}^{\infty} \frac{2}{\sqrt{2 \pi}} \exp \left(\frac{-u^{2}}{2}\right) d u \\
& =\frac{2(\sqrt{2})^{\delta}}{\sqrt{\pi}(\delta-2)} \Gamma\left(\frac{\delta+1}{2}\right) \text {. }
\end{aligned}
$$

Using the following result of Billingsley [3].

$$
\begin{aligned}
P\left(\sup _{0 \leq s \leq 1}|W(s)| \geq x\right) & =1-\sum_{k=-\infty}^{\infty}(-1)^{k} P((2 k-1) x \leq N \leq(2 k+1) x) \\
& =2 \sum_{k=0}^{\infty}(-1)^{k} P(|N| \geq(2 k+1) x),
\end{aligned}
$$

one can obtain (2.11).

Proposition 2.4. Under the conditions of Theorem 1.1, we have

$$
\begin{gathered}
\lim _{\epsilon \downarrow 0} \epsilon^{\delta-2} \sum_{n=2}^{\infty} \frac{1}{n^{2}(\log n)^{2 / \delta}} \mid \int_{\epsilon \sqrt{n}(\log n)^{1 / \delta}}^{\infty} 2 x P\left(\left|S_{n}\right| \geq x\right) d x \\
-\int_{\epsilon \sqrt{n}(\log n)^{1 / \delta}}^{\infty} 2 x P(|N| \geq x / \sqrt{n}) d x \mid=0,
\end{gathered}
$$


and

$$
\begin{gathered}
\lim _{\epsilon \downarrow 0} \epsilon^{\delta-2} \sum_{n=2}^{\infty} \frac{1}{n^{2}(\log n)^{2 / \delta}} \mid \int_{\epsilon \sqrt{n}(\log n)^{1 / \delta}}^{\infty} 2 x P\left(M_{n} \geq x\right) d x \\
-\int_{\epsilon \sqrt{n}(\log n)^{1 / \delta}}^{\infty} 2 x P\left(\sup _{0 \leq t \leq 1}|W(t)| \geq x / \sqrt{n}\right) d x \mid=0 .
\end{gathered}
$$

Proof. We only prove (2.12), let $H(\epsilon)=\left[\exp \left(M / \epsilon^{\delta}\right)\right], M>4,0<\epsilon<$ $1 / 4, \delta>2$, denote $\Delta_{n}=\sup _{x}\left|P\left(\left|S_{n}\right| \geq \sqrt{n} x\right)-P(|N| \geq x)\right|$, we have

$$
\begin{aligned}
\sum_{n=2}^{\infty} & \frac{1}{n^{2}(\log n)^{2 / \delta}} \mid \int_{\epsilon \sqrt{n}(\log n)^{1 / \delta}}^{\infty} 2 x P\left(\left|S_{n}\right| \geq x\right) d x \\
& -\int_{\epsilon \sqrt{n}(\log n)^{1 / \delta}}^{\infty} 2 x P(|N| \geq x / \sqrt{n}) d x \mid \\
= & \sum_{n \leq H(\epsilon)} \frac{1}{n^{2}(\log n)^{2 / \delta}} \mid \int_{\epsilon \sqrt{n}(\log n)^{1 / \delta}}^{\infty} 2 x P\left(\left|S_{n}\right| \geq x\right) d x \\
& -\int_{\epsilon \sqrt{n}(\log n)^{1 / \delta}}^{\infty} 2 x P(|N| \geq x / \sqrt{n}) d x \mid \\
& +\sum_{n>H(\epsilon)} \frac{1}{n^{2}(\log n)^{2 / \delta}} \mid \int_{\epsilon \sqrt{n}(\log n)^{1 / \delta}}^{\infty} 2 x P\left(\left|S_{n}\right| \geq x\right) d x \\
& -\int_{\epsilon \sqrt{n}(\log n)^{1 / \delta}}^{\infty} 2 x P(|N| \geq x / \sqrt{n}) d x \mid \\
= & : \Sigma_{1}+\Sigma_{2} .
\end{aligned}
$$

We first estimate $\Sigma_{1}$, it follows that

$$
\begin{aligned}
\Sigma_{1} \leq & \sum_{n \leq H(\epsilon)} \frac{1}{n}\left(\int_{(\log n)^{-1 / \delta} \Delta_{n}^{-1 / 4}}^{\infty} 2(x+\epsilon) P\left(|N| \geq(x+\epsilon)(\log n)^{1 / \delta}\right) d x\right. \\
& +\int_{0}^{(\log n)^{-1 / \delta} \Delta_{n}^{-1 / 4}} 2(x+\epsilon) \mid P\left(\left|S_{n}\right| \geq(x+\epsilon) \sqrt{n}(\log n)^{1 / \delta}\right) \\
& -P\left(|N| \geq(x+\epsilon)(\log n)^{1 / \delta}\right) \mid d x
\end{aligned}
$$




$$
\begin{aligned}
& \left.+\int_{(\log n)^{-1 / \delta} \Delta_{n}^{-1 / 4}}^{\infty} 2(x+\epsilon) P\left(\left|S_{n}\right| \geq(x+\epsilon) \sqrt{n}(\log n)^{1 / \delta}\right) d x\right) \\
= & \sum_{n \leq H(\epsilon)} \frac{1}{n}\left(\Sigma_{3}+\Sigma_{4}+\Sigma_{5}\right) .
\end{aligned}
$$

The estimate of $\Sigma_{3}$ is easy. By Toeplitz's lemma, one can complete the proof of term $\Sigma_{4}$. As to $\Sigma_{5}$, taking $a=\epsilon \sqrt{n}(\log n)^{1 / \delta}, b=a / m$ in Lemma 2.3, it turns out that

$$
\begin{aligned}
& \sum_{n \leq H(\epsilon)} \frac{\Sigma_{5}}{n} \leq C \sum_{n \leq H(\epsilon)} \frac{1}{n} \int_{(\log n)^{-1 / \delta} \Delta_{n}^{-1 / 4}}^{\infty} \frac{(y+\epsilon)^{-2 m+1} n^{-m}(\log n)^{-2 m / \delta}}{\left(m \sum_{k=1}^{n} E X_{k}^{2}\right)^{-m}} d y \\
& \quad+C \sum_{n \leq H(\epsilon)} \int_{(\log n)^{-1 / \delta} \Delta_{n}^{-1 / 4}}^{\infty}(y+\epsilon) P\left(\left|X_{1}\right| \geq(y+\epsilon) \sqrt{n}(\log n)^{1 / \delta} / m\right) d y \\
& =: \Sigma_{6}+\Sigma_{7} .
\end{aligned}
$$

An easy calculation leads to

$$
\begin{aligned}
\Sigma_{6} & \leq C \sum_{n \leq H(\epsilon)} \frac{1}{n(\log n)^{2 m / \delta}} \int_{(\log n)^{-1 / \delta} \Delta_{n}^{-1 / 4}}^{\infty}(y+\epsilon)^{-2 m+1} d y \\
& \leq C \frac{(\log H(\epsilon))^{1-2 / \delta}}{(\log H(\epsilon))^{1-2 / \delta}} \sum_{n \leq H(\epsilon)} \frac{1}{n(\log n)^{2 / \delta}} \Delta_{n}^{(m-1) / 2} \\
& =C \frac{\epsilon^{2-\delta} M^{1-2 / \delta}}{(\log H(\epsilon))^{1-2 / \delta}} \sum_{n \leq H(\epsilon)} \frac{1}{n(\log n)^{2 / \delta}} \Delta_{n}^{(m-1) / 2},
\end{aligned}
$$

by Toeplitz's lemma, we have $\lim _{\epsilon \downarrow 0} \epsilon^{\delta-2} \Sigma_{6}=0$. Turn to $\Sigma_{7}$, it follows that

$$
\begin{aligned}
\Sigma_{7} & \leq C \sum_{n \leq H(\epsilon)} E \int_{(\log n)^{-1 / \delta} \Delta_{n}^{-1 / 4}}^{\infty}(y+\epsilon) I\left(m(y+\epsilon) \leq\left|X_{1}\right| / \sqrt{n}(\log n)^{1 / \delta}\right) d y \\
& \leq C \sum_{n \leq H(\epsilon)} \frac{1}{n(\log n)^{2 / \delta}} E X_{1}^{2} I\left(m\left|X_{1}\right| \geq \sqrt{n}\right) \\
& =C \frac{(\log H(\epsilon))^{-2 / \delta+1}}{(\log H(\epsilon))^{-2 / \delta+1}} \sum_{n \leq H(\epsilon)} \frac{1}{n(\log n)^{2 / \delta}} E X_{1}^{2} I\left(m\left|X_{1}\right| \geq \sqrt{n}\right) \\
& \leq C \frac{\epsilon^{2-\delta} M^{1-2 / \delta}}{(\log H(\epsilon))^{-2 / \delta+1}} \sum_{n \leq H(\epsilon)} \frac{1}{n(\log n)^{2 / \delta}} E X_{1}^{2} I\left(m\left|X_{1}\right| \geq \sqrt{n}\right),
\end{aligned}
$$


applying Toeplitz's lemma again, we have $\lim _{\epsilon \downarrow 0} \epsilon^{\delta-2} \Sigma_{7}=0$. Now let us consider $\Sigma_{2}$, notice that

$$
\begin{aligned}
\Sigma_{2} \leq & C \sum_{n>H(\epsilon)} \frac{1}{n^{2}(\log n)^{2 / \delta}} \int_{\epsilon \sqrt{n}(\log n)^{1 / \delta}}^{\infty} 2 x P(|N| \geq x / \sqrt{n}) d x \\
& +C \sum_{n>H(\epsilon)} \frac{1}{n^{2}(\log n)^{2 / \delta}} \int_{\epsilon \sqrt{n}(\log n)^{1 / \delta}}^{\infty} 2 x P\left(\left|S_{n}\right| \geq x\right) d x \\
=: & \Sigma_{8}+\Sigma_{9} .
\end{aligned}
$$

Applying Markov's inequality, one can complete the estimate of $\Sigma_{8}$. By Lemma 2.3, taking $m>\delta / 2$, it turns out

$$
\begin{aligned}
\Sigma_{9} \leq & C \sum_{n>H(\epsilon)} \frac{1}{n} \int_{0}^{\infty} \frac{(y+\epsilon)^{-2 m+1} n^{-m}(\log n)^{-2 m / \delta}}{\left(m \sum_{k=1}^{n} E X_{k}^{2}\right)^{-m}} d y \\
& +C \sum_{n>H(\epsilon)} \int_{0}^{\infty}(y+\epsilon) P\left(\left|X_{1}\right| \geq(y+\epsilon) \sqrt{n}(\log n)^{1 / \delta} / m\right) d y \\
\leq & C \sum_{n>H(\epsilon)} \frac{1}{n(\log n)^{2 m / \delta}} \int_{0}^{\infty}(y+\epsilon)^{-2 m+1} d y \\
& +C E\left(\int_{0}^{\infty}(y+\epsilon) \sum_{n>H(\epsilon)} I\left(\sqrt{n}(\log n)^{1 / \delta} \leq m\left|X_{1}\right| /(y+\epsilon) d y\right)\right. \\
\leq & C \epsilon^{-2 m+2} \sum_{n>H(\epsilon)} \frac{1}{n(\log n)^{2 m / \delta}} \\
& +C E\left(\int_{0}^{\infty} \frac{X_{1}^{2}}{(y+\epsilon)}\left(\log \left|X_{1}\right|-\log (y+\epsilon)\right)^{-2 / \delta} I\left(y+\epsilon \leq m\left|X_{1}\right|\right) d y\right) \\
\leq & C \epsilon^{2-\delta} M^{-2 m / \delta+1}+C E X_{1}^{2}\left(\left(\log \left|X_{1}\right|\right)^{1-2 / \delta}-(\log \epsilon)^{1-2 / \delta}\right), \quad \quad(2.16)
\end{aligned}
$$

we have $\lim _{\epsilon \downarrow 0} \epsilon^{\delta-2} \Sigma_{9}=0$. The proof of (2.12) is now complete.

Proof of Theorem 1.1. Combining Propositions $2.1 \sim 2.4$, we have

$$
\lim _{\epsilon \downarrow 0} \epsilon^{\delta} \sum_{n=2}^{\infty} \frac{1}{n} P\left(\left|S_{n}\right| \geq \epsilon \sqrt{n}(\log n)^{1 / \delta}\right)=\frac{(\sqrt{2})^{\delta}}{\sqrt{\pi}} \Gamma\left(\frac{\delta+1}{2}\right),
$$




$$
\begin{gathered}
\lim _{\epsilon \downarrow 0} \epsilon^{\delta-2} \sum_{n=2}^{\infty} \frac{1}{n^{2}(\log n)^{2 / \delta}} \int_{\epsilon \sqrt{n}(\log n)^{1 / \delta}}^{\infty} 2 x P\left(\left|S_{n}\right| \geq x\right) d x \\
=\frac{2(\sqrt{2})^{\delta}}{\sqrt{\pi}(\delta-2)} \Gamma\left(\frac{\delta+1}{2}\right) .
\end{gathered}
$$

Then, the proof of (1.4) follows from (2.17) and (2.18), similarly, one can obtain (1.5).

For the sufficient part, using the standard method, we first show that

$$
P\left(\max _{1 \leq k \leq n}\left|X_{k}\right| \geq \epsilon \sqrt{n}(\log n)^{1 / \delta}\right) \rightarrow 0 \text { as } n \rightarrow \infty .
$$

It is easy to see that

$$
\left|X_{n}\right|=\left|S_{n}-S_{n-1}\right| \leq\left|S_{n}\right|+\left|S_{n-1}\right|,
$$

furthermore, we have

$$
\begin{gathered}
\left(\max _{1 \leq k \leq n}\left|X_{k}\right| \geq 2 \epsilon \sqrt{n}(\log n)^{1 / \delta}\right) \\
\subset\left(\max _{1 \leq k \leq n}\left|S_{k}\right| \geq \epsilon \sqrt{n}(\log n)^{1 / \delta}\right) \cup\left(\max _{1 \leq k \leq n-1}\left|S_{k}\right| \geq \epsilon \sqrt{n}(\log n)^{1 / \delta}\right) .
\end{gathered}
$$

Recalling (1.5) and (2.4), which yields

$$
\begin{aligned}
\infty & >\sum_{n=2}^{\infty} \frac{1}{n} P\left(\max _{1 \leq k \leq n}\left|X_{k}\right| \geq \epsilon \sqrt{n}(\log n)^{1 / \delta}\right) \\
& \geq \frac{C}{2} \sum_{m=2}^{\infty} P\left(\max _{1 \leq k \leq 2^{m}}\left|X_{k}\right| \geq \epsilon \sqrt{2^{m}}\left(\log 2^{m}\right)^{1 / \delta}\right) .
\end{aligned}
$$

Hence, we have

$$
P\left(\max _{1 \leq k \leq 2^{m}}\left|X_{k}\right| \geq \epsilon \sqrt{2^{m}}\left(\log 2^{m}\right)^{1 / \delta}\right) \rightarrow 0,
$$

So (2.19) follows from (2.22) and (2.23). We next show that

$$
P\left(\max _{1 \leq k \leq n}\left|X_{k}\right| \geq \epsilon \sqrt{n}(\log n)^{1 / \delta}\right) \geq C \sum_{k=1}^{n} P\left(\left|X_{k}\right| \geq \epsilon \sqrt{n}(\log n)^{1 / \delta}\right) .
$$


By the result of Joag-Dev et al. [7], it turns out that

$$
\begin{aligned}
& P\left(\max _{1 \leq k \leq n}\left|X_{k}\right| \geq \epsilon \sqrt{n}(\log n)^{1 / \delta}\right) \\
& \geq 1-\prod_{k=1}^{n}\left(1-P\left(\left|X_{k}\right| \geq \epsilon \sqrt{n}(\log n)^{1 / \delta}\right)\right) \\
& \quad \geq 1-\exp \left(-\sum_{k=1}^{n} P\left(\left|X_{k}\right| \geq \epsilon \sqrt{n}(\log n)^{1 / \delta}\right)\right),
\end{aligned}
$$

by (2.19), for sufficiently large $n$, we have

$$
\sum_{k=1}^{n} P\left(\left|X_{k}\right| \geq \epsilon \sqrt{n}(\log n)^{1 / \delta}\right) \rightarrow 0 \text { for any } \epsilon>0 .
$$

By (2.26) and using elementary inequality, we have

$$
\begin{aligned}
& P\left(\max _{1 \leq k \leq n}\left|X_{k}\right| \geq \epsilon \sqrt{n}(\log n)^{1 / \delta}\right) \\
& \quad \geq \sum_{k=1}^{n} P\left(\left|X_{k}\right| \geq \epsilon \sqrt{n}(\log n)^{1 / \delta}\right)\left(1-\sum_{k=1}^{n} P\left(\left|X_{k}\right| \geq \epsilon \sqrt{n}(\log n)^{1 / \delta}\right)\right) \\
& \quad \geq C \sum_{k=1}^{n} P\left(\left|X_{k}\right| \geq \epsilon \sqrt{n}(\log n)^{1 / \delta}\right) .
\end{aligned}
$$

Finally, the sufficient part follows from (2.27) together with

$$
\begin{aligned}
\infty & >\sum_{n=2}^{\infty} \frac{1}{n} \int_{0}^{\infty} 2(x+1) P\left(M_{n} \geq(x+1) \sqrt{n}(\log n)^{1 / \delta}\right) d x \\
& \geq C \sum_{n=N}^{\infty} \int_{0}^{\infty}(x+1) P\left(\left|X_{1}\right| \geq 2(x+1) \sqrt{n}(\log n)^{1 / \delta}\right) d x \\
& \geq C E\left(\int_{0}^{\infty}(y+\epsilon) \sum_{n>H(\epsilon)} I\left(\sqrt{n}(\log n)^{1 / \delta} \leq m\left|X_{1}\right| /(y+\epsilon) d y\right)\right. \\
& \geq C E\left(\int_{0}^{\infty} \frac{X_{1}^{2}}{(y+\epsilon)}\left(\log \left|X_{1}\right|-\log (y+\epsilon)\right)^{-2 / \delta} I\left(y+\epsilon \leq m\left|X_{1}\right|\right) d y\right) \\
& \geq C E X_{1}^{2}\left(\log \left|X_{1}\right|\right)^{1-2 / \delta} .
\end{aligned}
$$




\section{Proof of Theorem 1.2}

According to the proof of Theorem 1.1, we only give the main ideas of the proofs of (1.6) and (1.7). Observe (2.4) and (2.5), it is natural to give the following Propositions.

Proposition 3.1. We have

$$
\lim _{\epsilon \downarrow 0} \epsilon^{2 \delta+2} \sum_{n=2}^{\infty} \frac{(\log n)^{\delta}}{n} P\left(\left|S_{n}\right| \geq \epsilon \sqrt{n \log n}\right)=\frac{2^{\delta+1}}{\sqrt{\pi}(\delta+1)} \Gamma\left(\delta+\frac{3}{2}\right),
$$

and

$$
\begin{gathered}
\lim _{\epsilon \downarrow 0} \epsilon^{2 \delta+2} \sum_{n=2}^{\infty} \frac{(\log n)^{\delta}}{n} P\left(M_{n} \geq \epsilon \sqrt{n \log n}\right) \\
=\frac{2^{\delta+2} \Gamma\left(\delta+\frac{3}{2}\right)}{\sqrt{\pi}(\delta+1)} \sum_{n=0}^{\infty} \frac{(-1)^{n}}{(2 n+1)^{2 \delta+2}} .
\end{gathered}
$$

Proof. By a careful calculation, it follows that

$$
\lim _{\epsilon \downarrow 0} \epsilon^{2 \delta+2} \sum_{n=2}^{\infty} \frac{(\log n)^{\delta}}{n} P(|N| \geq \epsilon \sqrt{\log n})=\frac{2^{\delta+1}}{\sqrt{\pi}(\delta+1)} \Gamma\left(\delta+\frac{3}{2}\right) .
$$

Then, along the same lines as those of the proof of Proposition 2.2, we have

$$
\lim _{\epsilon \downarrow 0} \epsilon^{2 \delta+2} \sum_{n=2}^{\infty} \frac{(\log n)^{\delta}}{n}\left|P\left(\left|S_{n}\right| \geq \epsilon \sqrt{n \log n}\right)-P(|N| \geq \epsilon \sqrt{\log n})\right|=0 .
$$

With the help of Billingsley's result, one can complete the proof of (3.2).

Proposition 3.2. Under the conditions of Theorem 1.2, we have

$$
\begin{gathered}
\lim _{\epsilon \downarrow 0} \epsilon^{2 \delta} \sum_{n=2}^{\infty} \frac{(\log n)^{\delta-1}}{n^{2}} \int_{\epsilon \sqrt{n \log n}}^{\infty} 2 x P\left(\left|S_{n}\right| \geq x\right) d x \\
=\frac{2^{\delta+1}}{\sqrt{\pi} \delta(\delta+1)} \Gamma\left(\delta+\frac{3}{2}\right),
\end{gathered}
$$


and

$$
\begin{gathered}
\lim _{\epsilon \downarrow 0} \epsilon^{2 \delta} \sum_{n=2}^{\infty} \frac{(\log n)^{\delta-1}}{n^{2}} \int_{\epsilon \sqrt{n \log n}}^{\infty} 2 x P\left(M_{n} \geq x\right) d x \\
=\frac{2^{\delta+2} \Gamma\left(\delta+\frac{3}{2}\right)}{\sqrt{\pi} \delta(\delta+1)} \sum_{n=0}^{\infty} \frac{(-1)^{n}}{(2 n+1)^{2 \delta+2}} .
\end{gathered}
$$

Proof. Recalling the proof of Proposition 2.3, it is easy to get

$$
\begin{gathered}
\lim _{\epsilon \downarrow 0} \epsilon^{2 \delta} \sum_{n=2}^{\infty} \frac{(\log n)^{\delta-1}}{n^{2}} \int_{\epsilon \sqrt{n \log n}}^{\infty} 2 x P(|N| \geq x / \sqrt{n}) d x \\
=\frac{2^{\delta+1}}{\sqrt{\pi} \delta(\delta+1)} \Gamma\left(\delta+\frac{3}{2}\right) .
\end{gathered}
$$

The following proof is similar to that of Proposition 2.4, so we have

$$
\begin{gathered}
\lim _{\epsilon \downarrow 0} \epsilon^{2 \delta} \sum_{n=2}^{\infty} \frac{(\log n)^{\delta-1}}{n^{2}} \mid \int_{\epsilon \sqrt{n \log n}}^{\infty} 2 x P\left(\left|S_{n}\right| \geq x\right) d x \\
-\int_{\epsilon \sqrt{n \log n}}^{\infty} 2 x P(|N| \geq x / \sqrt{n}) d x \mid=0 .
\end{gathered}
$$

The moment condition $E X_{1}^{2}\left(\log \left|X_{1}\right|\right)^{\delta}<\infty$ is used as follows, note the corresponding part of $\Sigma_{9}$, we have

$$
\begin{aligned}
& C \sum_{n>H(\epsilon)} \frac{(\log n)^{\delta-1}}{n} \int_{\epsilon \sqrt{n \log n}}^{\infty} 2 x P\left(\left|X_{1}\right| \geq x\right) d x \\
& \leq C \sum_{n>H(\epsilon)}(\log n)^{\delta} \int_{0}^{\infty}(y+\epsilon) P\left(\left|X_{1}\right| \geq(y+\epsilon) \sqrt{n \log n} / m\right) d y \\
& \leq C E\left(\int_{0}^{\infty}(y+\epsilon) \sum_{n>H(\epsilon)}(\log n)^{\delta} I\left(\sqrt{n \log n} \leq m\left|X_{1}\right| /(y+\epsilon) d y\right)\right. \\
& \leq C E\left(\int_{0}^{\infty} \frac{X_{1}^{2}}{(y+\epsilon)}\left(\log \left|X_{1}\right|-\log (y+\epsilon)\right)^{\delta-1} I\left(y+\epsilon \leq m\left|X_{1}\right|\right) d y\right) \\
& \leq C E X_{1}^{2}\left(\left(\log \left|X_{1}\right|\right)^{\delta}-(\log \epsilon)^{\delta}\right),
\end{aligned}
$$


we then complete the proof of (3.5). Finally, observe that

$$
P\left(\sup _{0 \leq t \leq 1}|W(t)| \geq x\right) \leq 2 P(|N| \geq x)
$$

along the same proof lines of (3.5), one can complete the proof of (3.6).

Proof of Theorem 1.2. By virtue of Propositions 3.1 and 3.2, one can obtain (1.6) and (1.7). With the help of (2.27), the sufficient part is obvious.

\section{REFERENCES}

[1] K. Alam and K. M. L. Saxena, Positive dependence in multivariate distributions. Comm. Statist. A., 10 (1981), 1183-1196.

[2] R.E. Barlow and F. Proschan, Statistical theory of reliability and life testing: Probability Models. To begin with Publisher, Silver Spring, MD (1981).

[3] P. Billingsley, Convergence of probability measures. Wiley, New York (1968).

[4] J.T. Cox and G. Grimmett, Central limit theorems for associated random variables and the percolation model. Ann. Probab., 12 (1984), 514-528.

[5] K.A. Fu and L.X. Zhang, Precise rates in the law of the logarithm for negatively associated random variables. Comput. Math. Appl., 54 (2007), 687-698.

[6] A. Gut and A. Spătaru, Precise asymptotics in the Baum-Katz and Davis law of large numbers. J. Math. Anal. Appl., 248 (2000), 233-246.

[7] K. Joag-Dev and F. Proschan, Negative association of random variables with applications. Ann. Statist., 11 (1983), 286-295.

[8] H.Y. Liang, Complete convergence for weighted sums of negatively associated random variables. Statist. Probab. Lett., 48 (2000), 317-325.

[9] H.Y. Liang and C. Su, Complete convergence for weighted sums of NA sequences. Statist. Probab. Lett., 45 (1999), 85-95.

[10] L.X. Liu and R. Wu, Inequality of maximal partial sum and the law of iterated logarithm for sequences of NA random variables. Acta Math. Sinica., 45 (2002), 969-978.

[11] W.D. Liu and Z.Y. Lin, Precise asymptotics for a new kind of complete moment convergence. Statist. Probab. Lett., 76 (2006), 1787-1799.

[12] P. Matula, A note on the almost sure convergence of sums of negatively dependent random variables. Statist. Probab. Lett., 15 (1992), 209-213.

[13] C.M. Newman, Asymptotic independent and limit theorems for positively and negatively dependent random variables. In: Tong Y.L. (Ed.), Inequalities in Statistics and Probability. IMS. Hayward, CA (1984), 127-140. 
[14] V.V. Petrov, Limit theorems of probability theory-sequences of independent random variables. Oxford Science Publications, Oxford (1995).

[15] C.G. Roussas, Exponential probability inequalities with some applications. In: Ferguson T.S., Shapley L.S. and MacQueen J.B. (Ed.), Statistics, Probability and Game Theory. IMS. Hayward, CA (1996), 303-319.

[16] Q.M. Shao and C. Su, The law of the the iterated logarithm for negatively associated random variables. Stochastic Process. Appl., 83 (1999), 139-148.

[17] Q.M. Shao, A comparison theorem on maximum inequalities between negatively associated sequences and independent random variables. J. Theor. Probab., 13 (2000), 343-356.

[18] C. Su, L.C. Zhao and Y.B. Wang, The moment inequalities and weak convergence for negatively associated sequences. Science in China Series A. 39 (1996), 1091-1099.

[19] L.X. Zhang, The weak convergence for functions of negatively associated random variables. J. Multiv. Anal., 78 (2001), 272-298.

[20] L.X. Zhang and J.W. Wen, A weak convergence for negatively associated fields. Statist. Probab. Lett., 53 (2001), 259-267.

[21] Y. Zhao, Precise asymptotics for partial sums of nonstationary NA sequences. Acta Math. Sinica., 50 (2007), 539-546.

[22] Y. Zhao, Precise Rates in Log Laws for NA Sequences. Discrete Dyn. Nat. Soc., doi:10.1155/2007/89107: (2007), 11 pages. 